\title{
Resonant Rutherford Backscattering Studies of Cerium Oxide Thin Films Deposited by RF Sputtering
}

\author{
C.C. Chin \\ Institute of Astronomy and Astrophysics, Academia Sinica, Taiwan \\ R.J. Lin \\ Department of material Science, National Tsing Hua University, Taiwan \\ Y.C. Yu, C.W. Wang, E.K. Lin \\ Institute of Physics, Academia Sinica, Taiwan \\ W.C. Tsai and T.Y. Tseng \\ Department of Electronic Engineering, National Chiao Tung University, Taiwan
}

\begin{abstract}
We have studied the stoichiometry of cerium oxide films deposited by $\mathrm{RF}$ sputtering on sapphire and $\mathrm{MgO}$ as a function of deposition conditions using the resonant Rutherford backscattering method. We found that some films have the off-stoichiometry of $\mathrm{CeO}_{y}$ with $\mathrm{y}$ greater than 2.0. Such an off-stoichiometry cannot be due to a mixture of the known phases of bulk cerium oxide samples. This may be due to either cerium vacancies or interstitial oxygen atomic impurities. The cerium ion x-ray photoemission spectra of those films cannot determine the vacancy of the cerium ions. The c-axis $\mathrm{YBaCuO}$ thin flims deposited by sputtering on the $\mathrm{CeO}_{3.3}$ buffer layer on saphhire was found to be epitaxial. The $T_{c}$ was $86 \mathrm{~K}$ with $\Delta T_{c}$ less than $1 \mathrm{~K}$.
\end{abstract}

\section{INTRODUCTION}

$\mathrm{YBa}_{2} \mathrm{Cu}_{3} \mathrm{O}_{\mathrm{x}}$ thin films have been grown on cerium oxide $\mathrm{CeO}_{2}$ buffer layers on both sapphire and $\mathrm{Si}$ substrates by vacuum evaporation [1], laser ablation [2] and sputtering [3]. Several phases of $\mathrm{CeO}_{2}, \mathrm{Ce}_{2} \mathrm{O}_{3}$ and oxygen deficient $\mathrm{CeO}_{y}$ with $1.5<y<2.0[4,5]$ of bulk cerium oxide are known. Cerium oxide thin films may not have the stoichiometry of $\mathrm{CeO}_{2}$.

We have utilized resonant Rutherford Backscattering Spectrometry (RBS) to measure the oxygen contents of the cerium oxide thin films grown by on axis radio frequency (OARF) sputtering. We found that the stoichiometry depended on the sputtering conditions. Some samples have y $>2.0$. The $y$ should be equal to or less than 2.0 for all the known phases of bulk cerium oxides. This cannot be due to a mixture of the known phases of bulk samples. Those thin films with $y>2.0$ may either have cerium vacancies or oxygen interstitial defects. We have used the x-ray photoemission spectroscopy (XPS) to determine the valence of $\mathrm{Ce}$ ions from some selected samples. We have deposited $\mathrm{YBa}_{2} \mathrm{Cu}_{3} \mathrm{O}_{\mathrm{x}}$ thin film on a cerium oxide buffer layer with $\mathrm{y}$

Manuscript received August 27, 1996. equal to 3.3 to study how the value of $y$ affects on the growth of $\mathrm{YBa}_{2} \mathrm{Cu}_{3} \mathrm{O}_{\mathrm{x}}$ thin film.

\section{EXPERIMENTAL}

The $\mathrm{CeO}_{\mathrm{y}}$ samples were grown by two different deposition chambers $\mathrm{A}$ and $\mathrm{B}$ to confirm the universality of the off-stoichiometry. Table I summarizes the sample names, deposition conditions, and properties of the films. The RF power used in the chambers $A$ and $B$ was 60 and $40 \mathrm{~W}$ respectively. After deposition, the samples were cooled to room temperature in an atmospheric pressure of air, and atomsphere of 50 Torr of oxygen for chambers $\mathrm{A}$ and $\mathrm{B}$, respectively.

The $\mathrm{He}^{+}$ion beam was incident at an angle of a few degrees to the normal of the film to take the random RBS spectra. The detector was set at a laboratory scattering angle of 170 degrees. The energies of the channel numbers were calibrated by the Si and Au edges of the random spectra of a quartz crystal and a thin gold film grown on glass. All the samples were covered by a $5 \sim 10 \mathrm{~nm}$ thick Au layer to avoid charge up. The exact energy of the $\mathrm{He}^{+}$ions determined by the energy of the resonant RBS oxygen peak was $3.04 \mathrm{MeV}$. The thickness of our samples is $100 \mathrm{~nm}$, and therefore the resonance occurs near the surface of the samples. The stoichiometry of the $\mathrm{CeO}_{y}$ films was deduced from the fitting using program Gisa 3.91 [7]. The accurate enhanced oxygen cross sections from ref. [6] were used in the simulation.

The XPS spectra were acquired by the VG Microlab MK III system using the $\mathrm{Mg} \mathrm{Ka}$ X-ray radiation. The exposure time of the $x$-ray radiation on the sample was limited to a short period of 5 minutes [8] to avoid radiation damage.

The $\mathrm{YBa}_{2} \mathrm{Cu}_{3} \mathrm{O}_{\mathrm{x}}$ thin film was deposited by dc sputtering on a $\mathrm{CeO}_{y}$ buffer layer on sapphire (sample A5) at a substrate temperature of $700{ }^{\circ} \mathrm{C}$. The total chamber pressure was 200 mTorr while the $\mathrm{O}_{2} / \mathrm{Ar}$ ratio was 1:4. The dc sputtering current was $150 \mathrm{~mA}$. The orientations and the rocking curves 
of the $\mathrm{CeO}_{y}$ and $\mathrm{YBa}_{2} \mathrm{Cu}_{3} \mathrm{O}_{x}$ films were determined from $\mathrm{x}$-ray diffraction methods.

III. RESULTS

\section{A. RBS}

The fitting of the computer simulation to the random RBS spectrum of magnesium oxide substrate standard yields a stoichiometry of $\mathrm{MgO}$. It serves as the calibration of our measurement procedures, enhanced oxygen cross sections and the computer simulation.

Figures 1 and 2 show the examples of the measured random spectrum and the computer simulation of the samples A1 and A5, respectively. Although there are some scattering of the RBS data, the computer simulation fits well to both the cerium and resonant oxygen peaks. The stoichiometry of sample $\mathrm{A} 1$ was determined to be $\mathrm{Ce}_{1.0} \mathrm{O}_{2.022 .2}$ which was very close to $\mathrm{CeO}_{2}$. However, sample $\mathrm{A} 5$ was found to be $\mathrm{Ce}_{1.0} \mathrm{O}_{3.3}$. Figures 2 (b) and (c) show the details of the fitting to the oxygen and cerium peaks, respectively. The computer simulation of the stoichiometry of $\mathrm{Ce}_{1.0} \mathrm{O}_{2.0}$ was plotted as the dashed line in figures 2 (a), (b) and (c) for comparison. Table I summarizes the stoichiometry of all the samples deduced by the best fitting. The stoichiometries of the films depend sensitively on the deposition conditions as shown in Table I.

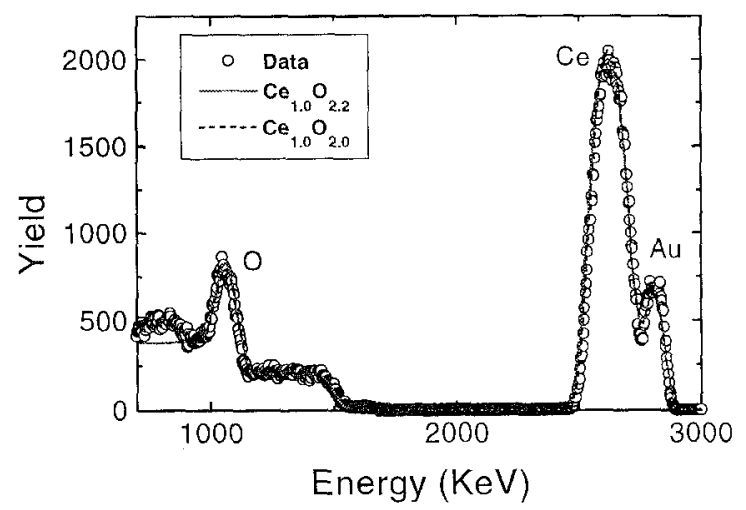

Fig.1 The random RBS spectrum and computer simulation of sample A1.

\section{B. XPS}

The energy of the cerium $3 d$ XPS spectrum of the thin films cannot match those of $\mathrm{CeO}_{2}$ or $\mathrm{Ce}_{2} \mathrm{O}_{3}$ [9]. No XPS spectrum other than the stoichiometry of $\mathrm{CeO}_{2}, \mathrm{Ce}_{2} \mathrm{O}_{3}$ has been reported. We cannot determine the valence of the cerium ion by XPS spectra. No clear correlation between the energy of the peaks and the stoichiometry was observed either. This may indicate that the off-stoichiometry with $y>2.0$ is caused by interstitial oxygen or cerium vacancies defects in the thin films.
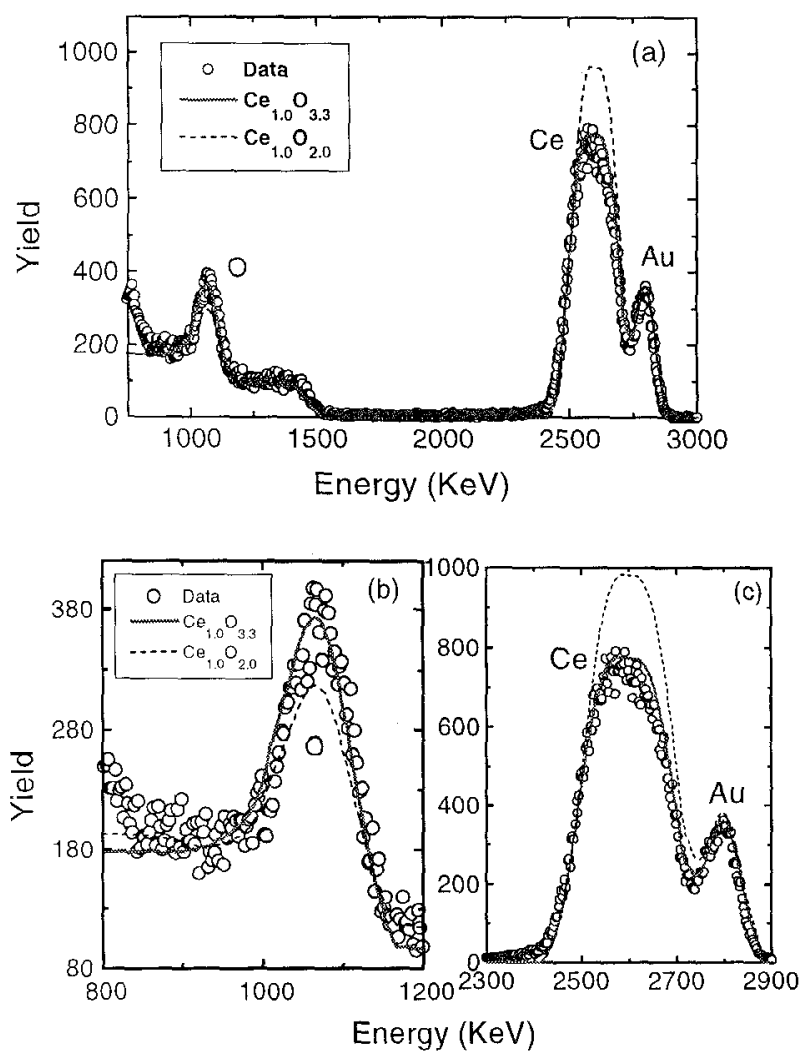

Fig. 2 (a) The random RBS spectrum and computer simulation of sample A5. (b) The enlarged view of the oxygen peak. (c) The enlarge view of the cerium peak.

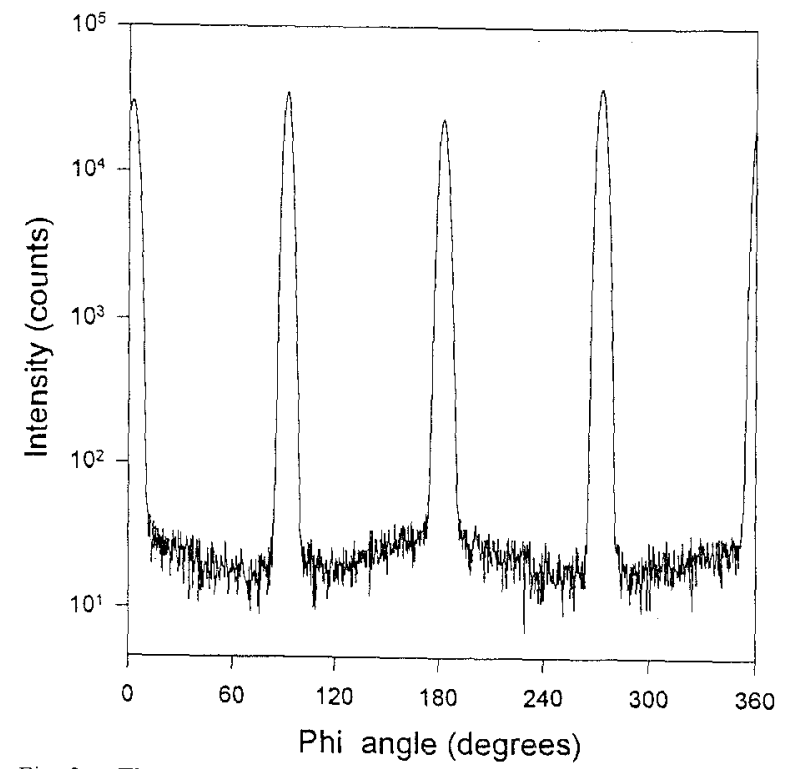

Fig. 3 The X-ray phi scan of the $(103)$ peaks of the

$\mathrm{YBa}_{2} \mathrm{Cu}_{3} \mathrm{O}$. films on $\mathrm{CeO}_{3}$ to indicate the 4-fold symmetry.

C. YBaCuO Film

$\mathrm{YBa}_{2} \mathrm{Cu}_{3} \mathrm{O}_{x}$ film grown on the top of the $\mathrm{CeO}_{3.3}$ (sample $\mathrm{A} 5$ ) as a c-axis orientation perpendicular to the substrate. The $T_{c}$ was $86 \mathrm{~K}$ with $\Delta T_{c}$ less than $1 \mathrm{~K}$. We get $T_{c}$ close to $90 \mathrm{~K}$ for films grown on $\mathrm{CeO}_{2}$ 
TABLE I

The deposition conditions and stoichiometries of the samples

\begin{tabular}{|c|c|c|c|c|c|c|c|c|}
\hline Sample & Chamber & Substrate & $\begin{array}{c}T_{d} \\
\left({ }^{\circ} \mathrm{C}\right)\end{array}$ & $\mathrm{O}_{2} / \mathrm{Ar}$ & $\begin{array}{c}\text { Deposition } \\
\text { Pressure } \\
\text { (mTorr) }\end{array}$ & $\begin{array}{l}\text { Orientation } \\
(111) /(200)\end{array}$ & $\begin{array}{c}\Delta \omega \\
\text { (Degree) }\end{array}$ & Stoichiometry \\
\hline Al & $\mathrm{A}$ & Sapphire & 720 & $5 / 100$ & 30 & $15 / 85$ & 4.67 & $\mathrm{Ce}_{1,0} \mathrm{O}_{2,(1)-2.2}$ \\
\hline $\mathrm{A} 2$ & $\mathrm{~A}$ & Sapphire & 720 & $25 / 100$ & 30 & $9 / 91$ & 2.68 & $\mathrm{Ce}_{1,0} \mathrm{O}_{2,5}$ \\
\hline A3 & A & Sapphire & 720 & $10 / 100$ & 30 & $9 / 91$ & 3.89 & $\mathrm{Ce}_{1.0} \mathrm{O}_{2.1122 .}$ \\
\hline A4 & A & Sapphire & 720 & $25 / 100$ & 20 & $98.4 / 1.6$ & $7.32^{*}$ & $\mathrm{Ce}_{1,0} \mathrm{O}_{1.8}$ \\
\hline A5 & $A$ & Sapphire & 720 & $5 / 100$ & 15 & $0 / 100$ & 0.59 & $\mathrm{Ce}_{1,0} \mathrm{O}_{3,3}$ \\
\hline A6 & $\mathrm{A}$ & $\mathrm{MgO}$ & 700 & $25 / 100$ & 15 & $11 / 89$ & 2.74 & $\mathrm{Ce}_{1,0} \mathrm{O}_{2.1}$ \\
\hline $\mathrm{A} 7$ & A & $\mathrm{MgO}$ & 750 & $25 / 100$ & 15 & $47 / 53$ & 3.76 & $\mathrm{Ce}_{1.0} \mathrm{O}_{2.4}$ \\
\hline A8 & A & $\mathrm{MgO}$ & 720 & $25 / 100$ & 15 & $2 / 98$ & 1.57 & $\mathrm{Ce}_{1.0} \mathrm{O}_{2.5}$ \\
\hline B1 & B & Sapphire & 600 & $100 / 100$ & 60 & $100 / 0$ & $0.31^{*}$ & $\mathrm{Ce}_{1,0} \mathrm{O}_{2,0 \sim 2,2}$ \\
\hline $\mathrm{B} 2$ & B & Sapphire & 760 & $100 / 100$ & 60 & $0 / 100$ & 0.3 & $\mathrm{Ce}_{1.0} \mathrm{O}_{3.3}$ \\
\hline $\mathrm{B} 3$ & $\mathrm{~B}$ & Sapphire & 800 & $100 / 100$ & 60 & $0 / 100$ & 0.3 & $\mathrm{Ce}_{1.0} \mathrm{O}_{1.1}$ \\
\hline B4 & B & Sapphire & 650 & $100 / 100$ & 100 & $91 / 9$ & 0.71 & $\mathrm{Ce}_{1,0} \mathrm{O}_{2.4}$ \\
\hline B5 & $\mathrm{B}$ & Sapphire & 710 & $100 / 100$ & 100 & $15 / 85$ & 0.29 & $\mathrm{Ce}_{1.0} \mathrm{O}_{1,9}$ \\
\hline
\end{tabular}

* Measured on the (111) peak.

The X-ray phi scan of the (103) peak shows the 4-folds symmetry as shown in figure 3 . It demonstrates that the in-plane orientation of the grains of $\mathrm{YBa}_{2} \mathrm{Cu}_{3} \mathrm{O}_{\mathrm{x}}$ films are epitaxial. It shows that epitaxial $\mathrm{YBa}_{2} \mathrm{Cu}_{3} \mathrm{O}_{\mathrm{x}}$ thin film can grow on $\mathrm{CeO}_{y}$ with a large value of y equal to 3.3 .

\section{DISCUSSION}

The sample and target holders were made of either Fe or Al. The oxides of those impurity atoms can cause y greater than 2.0. However, no $\mathrm{Fe}$ and $\mathrm{Al}$ impurity atom was observed in neither the RBS nor XPS spectra.

Most of the cerium isotopes have a nuclear mass of 140 and 142. The difference in the cross sections is very small. The energy of the $\mathrm{He}^{+}$ions scattered from these two isotopes should be within $5 \mathrm{KeV}$. This is much smaller than the resolution of the detector. Therefore, the usage of average Rutherford cross section and kinematic factor for the cerium atoms in the computer simulation should not affect the deduced stoichiometry of the sample. The cerium cross sections used were checked by fitting the non-resonant RBS spectrum of cerium oxide films reported in ref. [1]. The same stoichiometry close to $\mathrm{CeO}_{2.0}$ was obtained.

The cerium composition can be deduced by fitting only the height of the RBS spectrum of the cerium sublattice and the $\mathrm{Al}_{2} \mathrm{O}_{3}$ or $\mathrm{MgO}$ substrates. The oxygen composition can be obtained naturally afterward. Our computer simulation fits well to the cerium peaks. By fitting the RBS spectrum of $\mathrm{Ce}$ sublattice, the same oxygen contents were obtained as deduced by fitting the resonant oxygen peaks.

The stoichiometries of some selected samples were re-confirmed by fitting to the RBS data using the commerical program RMP. This confrimed the robustness of the program Gisa.

Such an off-stoichiometry cannot be due to the channeling or partial channeling effect. Firstly, although samples A5, B2, B4 which have the stoichiometry with $y>2$ have rather good 
crystallinity (small values of $\Delta \omega$ ), samples A2, A7 and A8 have very poor crystallinity. Channeling effect is impossible on those samples with poor crystallinity. Secondly, if any partial channeling effect occurs, both the cerium and oxygen peaks will decrease and then the spectra cannot be fitted to the computer simulation which assumes complete random scattering of the $\mathrm{He}^{+}$ions. Thirdly, the random spectra were repeated at different incident angles. The same spectra were obtained.

Therefore, the off-stoichiometry with $y$ greater than 2.0 should not be due to any embedded impurity atoms, wrong input resonant or non-resonant cross sections, computer errors or even channeling effect.

We cannot find a clear correlation between (200) and (111) lattice spacing with the stoichiometry. As discussed above, the valence of $\mathrm{Ce}$ ions of those samples with $\mathrm{y}>2.0$ cannot match with the valence of $\mathrm{Ce}$ ions of existing phases. Both of these may indicate that the off-stoichiometry with $y>2.0$ is caused by interstitial oxygen or cerium vacancies defects in the thin films.

\section{Conclusion}

We found that the stoichiometry of cerium oxide thin film varied sensitively with the deposition conditions. Chin and Mortishita [9] have observed that the oxygen content of the a-axis $\mathrm{YBa}_{2} \mathrm{Cu}_{3} \mathrm{O}_{\mathrm{x}}$ oxide films deposited by $\mathrm{RF}$ sputtering depends sensitively on the deposition temperature. It may be a general phenomenon that the oxygen contents of oxide films depend on the deposition conditions.

Some cerium oxide films even have the $y>2.0$. This cannot be due to a mixture of the known phases of the bulk samples. It is not clear whether it is due to cerium vacancies or interstitial oxygen atoms. Future transmission electron microscopy study may help us to answer this question. Unfortunately, XPS studies cannot determine the valence of the cerium ion in those samples.

The c-axis $\mathrm{YBa}_{2} \mathrm{Cu}_{3} \mathrm{O}_{\mathrm{x}}$ film grown on the top of the $\mathrm{CeO}_{3.3}$ buffer layer was epitaxial. A $T_{c}$ of $86 \mathrm{~K}$ with $\Delta T_{c}$ less than $1 \mathrm{~K}$ was obtained. To understand how the stoichiometry affects the growth of $\mathrm{YBa}_{2} \mathrm{Cu}_{3} \mathrm{O}_{\mathrm{x}}$ film, detailed studies of the $\mathrm{YBa}_{2} \mathrm{Cu}_{3} \mathrm{O}_{\mathrm{x}}$ film grown on the top of the $\mathrm{CeO}_{\mathrm{y}}$ for different values of $y$ are now being carried out.

\section{REFERENCES}

[1] T. Inoue, Y. Yamamoto, S. Koyama, S. Suzuki and Y. Ueda, Appl. Phys. Lett., vol. 56, p.1332, 1990

[2] X.D. Wu, S.R. Foltyn, R.E. Muenchausen, D.W. Cooke, A. Pique, D. Kalokitis, V. Pendrick and E. Belohoubek, Journal of Superconductivity, vol. 5, p. 353,1992 .

[3] F. Wang and R. Wordenweber, Thin Solid Films, vol. 227, p.200, 1993.
[4] R. Korner, M. Ricken and J. Nolting, Jorunal of Solid State Chemistry, vol. 78, p. 136-147, 1989.

[5] O. Toft Sorensen, Journal of Solid State Chemistry, vol. 18, p. 217-233, 1976.

[6] J.A. Leavitt, L.C. Jr. Miclntyre, M.D. Ashbaugh, J.G. Oder, Z. Lin and B. Dezfouly-Arjomandy, Nucl. Instrum. Methods in Phys. Research B vol. 44 p.260, 1990

[7] I. Saarilahti and E. Rauhala, Nucl. Instrum. Methods in Phys. Research B vol 46, p. 734-738, 1992

[8] E. Paparazzo, G.M. Ingo and N. Zacchetti, J. Vac. Sci. Technol, vol. A9(3), p. 1416,1991

[9] C.C. Chin, and T.Morishita, Physica C, vol. 243, p.373, 1995. 